\title{
EDUCAÇÃO NUTRICIONAL PARA PRÉ-ESCOLARES: UMA FERRAMENTA DE INTERVENÇÃO
}

\author{
Mônica Cristina Lopes do Carmo ${ }^{1}$ \\ Luiza Carla Vidigal Castro ${ }^{2}$ \\ Juliana Farias de Novaes ${ }^{3}$
}

\begin{abstract}
RESUMO: A faixa etária pré-escolar caracteriza-se por um período de alta vulnerabilidade e susceptibilidade à má nutrição. Nesta fase, as carências nutricionais levam a um crescimento deficiente, aumento do risco de infecções; alterações no processo de maturação do sistema nervoso e no desenvolvimento mental e intelectual, provocando desequilíbrios funcionais. O presente artigo tem como objetivo traçar o perfil nutricional de pré-escolares em nove Núcleos Comunitários do município de Viçosa-MG, promovendo, neste período, a melhoria do estado nutricional por meio de educação nutricional. A metodologia utilizada caracteriza-se pela avaliação antropométrica, em que se aferiram peso e estatura para posterior análise dos índices (P/I), (P/E) e (E/I). Os resultados representam a avaliação de 155 pré-escolares antes da intervenção: 91,61\% $(n=142)$ das crianças apresentaram estado nutricional de eutrofia, 7,09\% $(\mathrm{n}=11)$ apresentaram sobrepeso, $1,29 \%(n=2)$ apresentaram baixo peso. Após a intervenção foi avaliado o estado nutricional de 95 crianças, com o seguinte diagnóstico: $94 \%(n=89)$ crianças eutróficas, $1 \%(n=1)$ crianças com baixo peso e $5 \%(n=5)$ crianças com sobrepeso. Portanto, é possível observar que a participação da comunidade escolar e a adequada formação de agentes envolvidos nos procedimentos de Segurança Alimentar para as Unidades de Alimentação e Nutrição Escolar são indispensáveis para a prática promotora de saúde de crianças institucionalizadas que recebem a merenda escolar.
\end{abstract}

PALAVRAS-CHAVE: Pré-escolares. Educação nutricional. Avaliação antropométrica. Creche.

Nutrition education for preschool: a tool for action

\begin{abstract}
The preschool age is characterized by a period of high vulnerability and susceptibility to malnutrition. In this phase, nutritional deficiencies lead to growth deficiency, increase of infection risk, changes in the maturation of the nervous system, mental and intellectual development, causing functional imbalances. This paper goals to trace the nutritional status of preschoolers in 9 Community Centers of the city Viçosa in the state of Minas Gerais, promoting, during this period, through nutrition education, improvement of nutritional status. Anthropometric evaluation has measured the weight and height for later analysis of the indexes $(\mathrm{P} / \mathrm{I}),(\mathrm{P} / \mathrm{E})$ and $(\mathrm{E} / \mathrm{I})$. The results represent the evaluation of 155 preschool before the intervention: $91.61 \%(\mathrm{n}=142)$ of the children had nutritional status of eutrophy, $7.09 \%(n=11)$ of them were overweight and $1.29 \%(n=2)$ were underweight. After the intervention, it was assessed the nutritional status of 95 children with the following diagnosis: 94\% $(n=89)$ eutrophic children, $1 \%(n=1)$ were underweight and $5 \%(n=5)$ were overweight

\footnotetext{
${ }^{1}$ Mestranda em Ciências da Nutrição e do Esporte e Metabolismo pela Universidade Estadual de Campinas (monica.carmo@ufv.br).

${ }^{2}$ Doutora em Ciência e Tecnologia de Alimentos pela Universidade Federal de Viçosa, professora no curso de Nutrição da Universidade Federal de Viçosa (luiza.castro@ufv.br).

${ }^{3}$ Doutora em Ciências da Saúde pela Universidade Federal de Minas Gerais, professora no curso de Nutrição da Universidade Federal de
} Viçosa (jnovaes@ufv.br).
\end{abstract}


children. Therefore, the participation of the educational community and proper training of staff involved with the procedures from Food Security to the Supply Units and Educational Nutrition are essential to promote health to institutionalized children who have school meals.

KEYWORDS: Preschoolers. Nutritional education. Anthropometric evaluation. Daycare.

\section{INTRODUÇÃO}

A educação tem por finalidade possibilitar o desenvolvimento das capacidades dos indivíduos em condição de aprendizagem, como componente de autorrealização, e não simplesmente "transmitir" conhecimentos de uma pessoa para outra. Assim, considerando-se que os hábitos e práticas de saúde são formados precocemente na vida, as crianças devem ser encorajadas a desenvolver atitudes de saúde positivas (AZEVEDO, 2013). Por isso, utilizar educação em saúde desde a infância é uma estratégia para diminuir os custos com doenças, pois, por meio da prevenção, é possível promover a saúde ainda na infância (SMELTZER; BARE, 2005).

Énesta fase do desenvolvimento que ocorre a consolidação e a formação dos hábitos alimentares, o que justifica a importância da educação nutricional, a fim de se obter a promoção da saúde e a prevenção de doenças crônicas não transmissíveis dos indivíduos na fase adulta (ALBIERO; ALVES, 2007).

O pré-escolar é um grupo etário que se caracteriza por um grande interesse pelo ambiente no qual se encontra inserido (LIMA et al., 2009). Neste sentido, a educação nutricional é de suma relevância, devendo consistir em processo ativo, lúdico e interativo.

A educação nutricional pode ser definida como "uma variedade de experiências planejadas, a fim de facilitar a adoção voluntária de hábitos alimentares ou de qualquer comportamento relacionado à alimentação, que conduz à saúde e ao bem estar" (FAGIOLI; NASSER, 2006, p. 211-218) Assim, o planejamento destas experiências deve levar em consideração as características específicas do grupo para o qual se destinam as ações.

Estudos têm despertado a atenção para as vantagens da análise do estado nutricional de crianças a partir do espaço/instituição que elas frequentam, como escolas e creches (BARROS; VICTORA; VAUGHAN, 1990; ANTONIO et al., 1996). O interesse a respeito do conhecimento da magnitude dos problemas nutricionais, tendo como unidade de diferenciação o espaço/instituição frequentado, reside na possibilidade da identificação da distribuição dos distúrbios nutricionais, do monitoramento das desigualdades sociais em saúde e, principalmente, de possibilitar a identificação de necessidades de implementação de ações específicas e diferenciadas de nutrição e saúde.

A faixa etária pré-escolar caracteriza-se por um período de crescimento lento, porém contínuo, de alta vulnerabilidade e susceptibilidade à má nutrição. Nesta fase da vida, as carências nutricionais levam a um crescimento deficiente, aumento do risco de infecções, alterações no processo de maturação do sistema nervoso, no desenvolvimento mental e intelectual, provocando desequilíbrios morfológicos e funcionais, os quais, dependendo da intensidade e duração, 
podem até ser irreversíveis, ou até mesmo resultar em mortalidade precoce (OLIVEIRA; SILVA; SANT'ANA, 2003).

O objetivo deste artigo, portanto, consiste relatar as ações de um projeto, que visou traçar o perfil nutricional de pré-escolares em nove Núcleos Comunitários do município de Viçosa-MG, promovendo neste período, por meio de educação nutricional, a melhoria do estado nutricional dessas crianças.

\section{METODOLOGIA}

O estudo foi do tipo longitudinal, constituído de levantamento de dados antropométricos em préescolares institucionalizados, no período de março a dezembro de 2010, em nove Núcleos Comunitários da Educação Infantil do município de Viçosa-MG, perfazendo um total de 186 crianças na faixa etária de dois a cinco anos de idade, de ambos os sexos, frequentadoras das creches da rede municipal.

A pesquisa foi realizada após consentimento dos órgãos competentes e aprovação do protocolo Comitê de Ética na Pesquisa com Seres Humanos da Universidade Federal de Viçosa, sob o número 014/2006. Os responsáveis pelas crianças foram informados do estudo e assinaram o Termo de Consentimento Livre Esclarecido.

$\mathrm{Na}$ avaliação antropométrica, foram adotadas as técnicas de Jelliffe (1966) para a obtenção do peso e estatura. Para a medida do peso foi utilizada balança portátil, digital, eletrônica, da marca Personal Scale, QE-2003A, com capacidade de 150kg e precisão de 100g. Tais medidas foram aferidas pelos mesmos dois avaliadores, com ampla experiência na realização de medidas antropométricas com essas características (um avaliador para realizar as medidas entre os meninos e outro avaliador para realizar as medidas entre as meninas).

Posteriormente, realizaram-se as análises dos índices peso para idade $(\mathrm{P} / \mathrm{I})$, peso para estatura $(\mathrm{P} / \mathrm{E})$ e estatura para idade (E/I), utilizando-se como critério diagnóstico o "escore-z" e adotando-se como referência antropométrica a Organização Mundial da Saúde (WHO, 2006). Foram diagnosticadas, como baixo peso, as crianças cujos índices P/I e P/E se encontraram abaixo de -2 "escore z" e, com sobrepeso, aquelas com peso/estatura superior a +2 "escore $\mathrm{z}$ ". As crianças que apresentaram o índice E/I abaixo de -2 "escore z" foram classificadas como de baixa estatura (OMS, 2006).

Após o diagnóstico, realizou-se uma reunião com os pais e/ou responsáveis para informá-los da classificação do estado nutricional dos pré-escolares, além de receberem orientações a respeito e esclarecerem dúvidas.

Foi realizado, no início do projeto, um curso para capacitação de manipuladores de alimentos, com duração de 4 horas. O curso intitulado "Capacitação para Colaboradores" abordou conteúdos relacionados ao processo de decisão na elaboração dos cardápios, com ênfase em per capita e porcionamento dos alimentos, desgaste no processo de trabalho das merendeiras e alimentação escolar como espaço para incorporação de hábitos alimentares saudáveis, o Programa Nacional de Alimentação Escolar (PNAE) e seus objetivos, a importância da merendeira no sistema de 
avaliação escolar, princípios de nutrição, princípios de boas práticas nas diversas etapas da produção de alimentos e receitas nutritivas.

A aula prática incluiu demonstrações e degustação de receitas com proteína texturizada de soja (PTS), porcionamento de alimentos de acordo com a Pirâmide Alimentar Infantil e preparo de tempero caseiro com condimentos naturais (BARBOSA; SALLES-COSTA; SOARES, 2006).

Foram capacitadassetemerendeiras, comidadevariandoentre $34 \mathrm{e} 66 \mathrm{anos}$, sendo todas do sexo feminino.

As reuniões com os professores dos núcleos comunitários da Educação Infantil ocorreram no final do projeto e abordaram os temas "O papel do educador na alimentação infantil" e "Os dez passos para uma alimentação saudável” (SCHMITZ et al., 2013; VITOLO et al., 2005). Além disso, foram apresentados os resultados do estudo e esclarecidas dúvidas em relação à alimentação infantil. Nessa palestra, utilizou-se como recurso didático um álbum seriado do Ministério da Saúde.

A fim de informar os pais e/ou responsáveis, professores e auxiliares a respeito de uma alimentação saudável na infância foi elaborada uma cartilha educativa, explicando sobre a Pirâmide Alimentar Infantil. Utilizou-se como recurso didático uma pirâmide alimentar feita de feltro e os alimentos de material plástico (Etileno Acetato de Vinila - E.V.A.).

Após as intervenções, as crianças foram novamente submetidas à avaliação antropométrica e dietética, a fim de verificar se houve melhora do estado nutricional.

A realização das apresentações lúdicas para as crianças contou com a interação dos professores, crianças e estudantes de Nutrição, fortalecendo, assim, a aprendizagem dos envolvidos.

Os resultados foram expressos em porcentagem, média e desvio padrão. Para a análise das variáveis, utilizou-se o teste $\mathrm{Z}$ para duas proporções. Considerou-se significante $\mathrm{p}<0,05$.

\section{RESULTADOS E DISCUSSÃO}

Todas as creches pertencentes ao estudo referiram ter atividades relacionadas à alimentação previstas em seus Projetos Político-Pedagógicos. É interessante destacar que, em um estudo realizado em 2004 para avaliar o Programa Nacional de Alimentação Escolar, foi apontado que 38,3\% das escolas públicas brasileiras realizaram alguma atividade de educação alimentar e nutricional, sendo que, no Estado de São Paulo, foi encontrado o melhor índice, 56,4\% (BRASIL, 2006).

A Tabela 1 apresenta a distribuição das crianças quanto ao gênero nos dois períodos de avaliação antropométrica (Figura 1). 
Tabela 1 - Distribuição dos pré-escolares antes e depois da intervenção de acordo com o gênero.

\begin{tabular}{lccccc}
\hline \multirow{2}{*}{ Tempo de Intervenção } & \multicolumn{5}{c}{ Gênero } \\
\cline { 2 - 6 } & $\mathrm{n}$ & \multicolumn{3}{c}{ Masculino } & \multicolumn{3}{c}{ Feminino } \\
\cline { 2 - 6 } & & $\mathrm{n}$ & $\%$ & $\mathrm{n}$ & $\%$ \\
\hline T1* & 155 & 82 & 52,9 & 73 & 47,1 \\
T2 $^{* *}$ & 95 & 51 & 53,68 & 44 & 46,32 \\
\hline
\end{tabular}

* Antes da intervenção

** Após a intervenção

Fonte: Elaborada pelas autoras.

Figura 1: Avaliação antropométrica de pré-escolares.
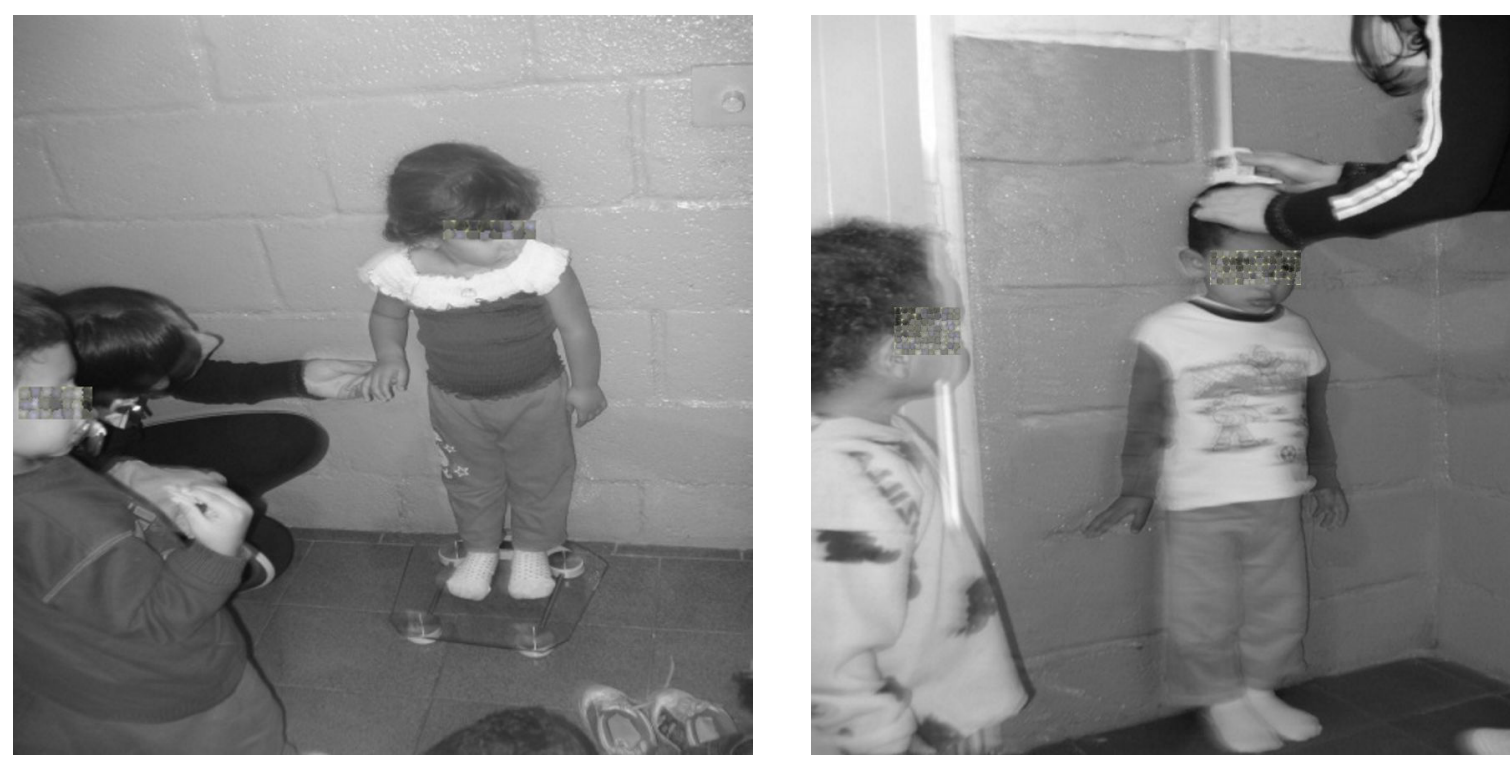

Fonte: Acervo das autoras.

A análise estatística dos índices antropométricos iniciais e finais de todas as crianças pode demonstrar o quão eficiente foi a metodologia desenvolvida.

Em relação ao estado nutricional das crianças, verificou-se antes da intervenção que a maioria das crianças apresentava estado nutricional de eutrofia, representando 91,61\% ( $n=142)$ do total. Entre o restante, 7,09\% $(n=11)$ das crianças se encontravam com sobrepeso, e 1,29\% $(\mathrm{n}=2)$ apresentavam-se com baixo peso. As variáveis, quando testadas com a análise estatística, mostraram-se significativas $(\mathrm{p}<0,05)$ (Tabela 2$)$.

Após a intervenção, foi avaliado o estado nutricional de 95 crianças, com o seguinte diagnóstico: 94\% $(n=89)$ de crianças eutróficas, $1 \%(n=1)$ delas com baixo peso e $5 \%(n=5)$ com sobrepeso (Tabela 2$)$. 
Tabela 2 - Classificação de do estado nutricional das crianças antes e após a intervenção. Viçosa, MG, 2010

\begin{tabular}{lcccc}
\hline \multirow{2}{*}{ Estado Nutricional } & \multicolumn{2}{c}{$\mathrm{T} 1$} & $\mathrm{n}$ & $\mathrm{T} 2$ \\
\cline { 2 - 5 } & $\mathrm{N}$ & $\%$ & 1 & 1 \\
\hline Baixo Peso & 2 & 1,29 & 89 & 94 \\
Eutrofia & 142 & 91,61 & 5 & 5 \\
Sobrepeso & 11 & 7,09 & 0 & 0 \\
Baixa Estatura & 0 & 0 & 09 \\
\hline
\end{tabular}

Fonte: Elaborada pelas autoras.

Assim, comparando as diferenças entre T1 e T2, a porcentagem de crianças eutróficas aumentou de $91,61 \%$ para $94 \%$ e a de crianças com baixo peso diminuiu de $1,29 \%$ para $1 \%$. Da mesma forma, o percentual de crianças com peso elevado para estatura e/ou idade também diminuiu, de $7,09 \%$ para $5 \%$. Esta diferença foi significante $(\mathrm{p}<0,001)$.

Nos dois períodos de avalição não foram detectadas crianças com baixa estatura. Este estudo, ao contemplar a avaliação do estado nutricional, ressalta a importância de uma alimentação apropriada na infância, para que lhes seja garantido um desenvolvimento normal, além do estabelecimento de um nível adequado de saúde, uma vez que se trata de um estágio de vida vulnerável aos distúrbios nutricionais e à ocorrência de doenças (GIUGLIANI; VICTORA, 2000).

Em estudo conduzido por Miranda e colaboradores, em 2003, em que se utilizou o índice peso/ idade para classificar o baixo peso em crianças assistidas pelo serviço público de saúde, também do município de Viçosa-MG, foram encontrados os valores de 11,7\% (MIRANDA et al., 2003).

Ribas e colaboradores (1999) observaram 3,2\% para o déficit de P/I e 6,3 para o de E/I, em crianças de 0 a 59 meses de uma população da região Centro-Oeste do Brasil. Corso, Viteritte e Peres (2004) encontraram uma prevalência de $1,1 \%$ de déficit de $\mathrm{P} / \mathrm{E}$, ao estudarem crianças menores de 6 anos de creches públicas de Florianópolis, SC; Tuma, Costa e Schmitz (2005) observaram prevalência de desnutrição de 4,8\%, 2,2\% e 0,4\% segundo os índices E/I, P/I e P/E respectivamente.

O déficit estatural não foi encontrado neste estudo e, portanto, foi substancialmente inferior ao registrado no país (10,4\%) e ao encontrado no inquérito nacional realizado em 1996 (7,7\%) nas regiões urbanas do país (MONTEIRO; CONDE, 2000).

A diminuição da prevalência de déficits antropométricos tem sido verificada em todo o país, em várias pesquisas, e atribuída a progressos moderados na renda familiar, à expansão da cobertura de serviços de saneamento, saúde e educação e à oferta de programas de suplementação alimentar (GALLO et al., 2000; MONTEIRO, 2008).

No estudo de Monteiro e Conde (2000) foi apontada a prevalência de 2,4\% para o déficit de E/I e 0,6\% para o de P/E em crianças do Estado de São Paulo. Já Queiroz (2008), em estudo populacional realizado na Paraíba, encontrou, em crianças entre 6 e 59 meses de idade, 3,3\%, $4,9 \%$ e $0,8 \%$ para os índices, $\mathrm{P} / \mathrm{I}, \mathrm{E} / \mathrm{I}, \mathrm{P} / \mathrm{E}$, respectivamente. 
Os resultados encontrados no presente estudo assemelham-se com publicações recentes, que indicam que o Brasil tem apresentado mudanças no perfil nutricional de sua população, processo esse chamado de transição nutricional (MONTEIRO et al., 2008; ZAMBOM et al., 2003).

Avaliando as variáveis "peso" e "estatura", o gênero masculino teve como mediana observada para peso $15,05 \mathrm{~kg}$, em T1 e em T2, 16,7kg, sendo a diferença entre T2 e T1 de 1,65kg, o que superou o ganho de peso esperado, de acordo com a população de referência (WHO, 2006). Da mesma forma, no gênero feminino, observou-se um ganho de peso em relação às medianas de $1,7 \mathrm{~kg}$ superior ao esperado (Tabela 3 ).

Tabela 3 - Mediana da variável peso, avaliada de acordo com o observado e esperado em cada gênero.

\begin{tabular}{lcccccc}
\hline & \multicolumn{6}{c}{ Peso $(\mathrm{Kg})$} \\
\cline { 2 - 6 } Gênero & Mediana & Observada & Diferença & Mediana Esperada* & Diferença \\
\hline \multirow{2}{*}{ Masculino } & T1 & T2 & & T1 & T2 & \\
Feminino & 15,05 & 16,7 & 1,65 & 14,3 & 15 & 0,7 \\
& 14,2 & 15,9 & 1,7 & 13,9 & 14,6 & 0,7 \\
\hline
\end{tabular}

*Mediana esperada mostram os valores no p50 da população de referência para mesma idade e gênero (WHO, 2006).

Fonte: Elaborada pelas autoras.

Em relação à estatura em T1, a mediana observada no gênero masculino foi de $99 \mathrm{~cm}$ e $101,2 \mathrm{~cm}$ em $\mathrm{T} 2$, sendo a diferença de $2,2 \mathrm{~cm}$, ligeiramente inferior ao esperado $(2,5 \mathrm{~cm})$. Já no gênero feminino, o ganho estatural observado foi igual ao esperado. Esta diferença foi significante $(p<0,001)$ (Tabela 4$)$.

Tabela 4 - Mediana da variável estatura, avaliada de acordo com o observado e esperado em cada gênero.

\begin{tabular}{|c|c|c|c|c|c|c|}
\hline \multirow{3}{*}{ Gênero } & \multicolumn{6}{|c|}{ Estatura $(\mathrm{cm})$} \\
\hline & \multicolumn{2}{|c|}{ Mediana Observada } & \multirow[t]{2}{*}{ Diferença } & \multicolumn{2}{|c|}{ Mediana Esperada } & \multirow[t]{2}{*}{ Diferença } \\
\hline & $\mathrm{T} 1$ & $\mathrm{~T} 2$ & & $\mathrm{~T} 1$ & $\mathrm{~T} 2$ & \\
\hline Masculino & 99 & 101,2 & 2,2 & 96,1 & 98,6 & 2,5 \\
\hline Feminino & 96,2 & 98,8 & 2,6 & 95,1 & 97,7 & 2,6 \\
\hline
\end{tabular}

Fonte: Elaborada pelas autoras.

Estes resultados despertam interesse quanto à possibilidade de adequação das merendas escolares, considerando-se os diferentes padrões nutricionais prevalentes no interior da rede pública de ensino, apontando para a necessidade das atividades de vigilância nutricional, atuação articulada escolas/serviços de saúde para o monitoramento dos perfis nutricionais e intervenções diferenciadas para os distintos espaços/instituições frequentados pelos escolares.

O uso de atividades lúdicas como forma de estratégica para a construção do conhecimento arregimenta uma nova postura existencial, cujo paradigma é um novo sistema de aprender brincando, inspirado em uma concepção de educação para além da instrução (MONTEIRO et al., 2008). Pode-se observar que as crianças apresentavam grande interesse em expressar suas opiniões, gostos, hábitos, preferências e experiências alimentares, interagindo com as brincadeiras (Figura 1). 
Figura 2 - Educação nutricional para pré-escolares dos Núcleos Comunitários de Viçosa-MG.
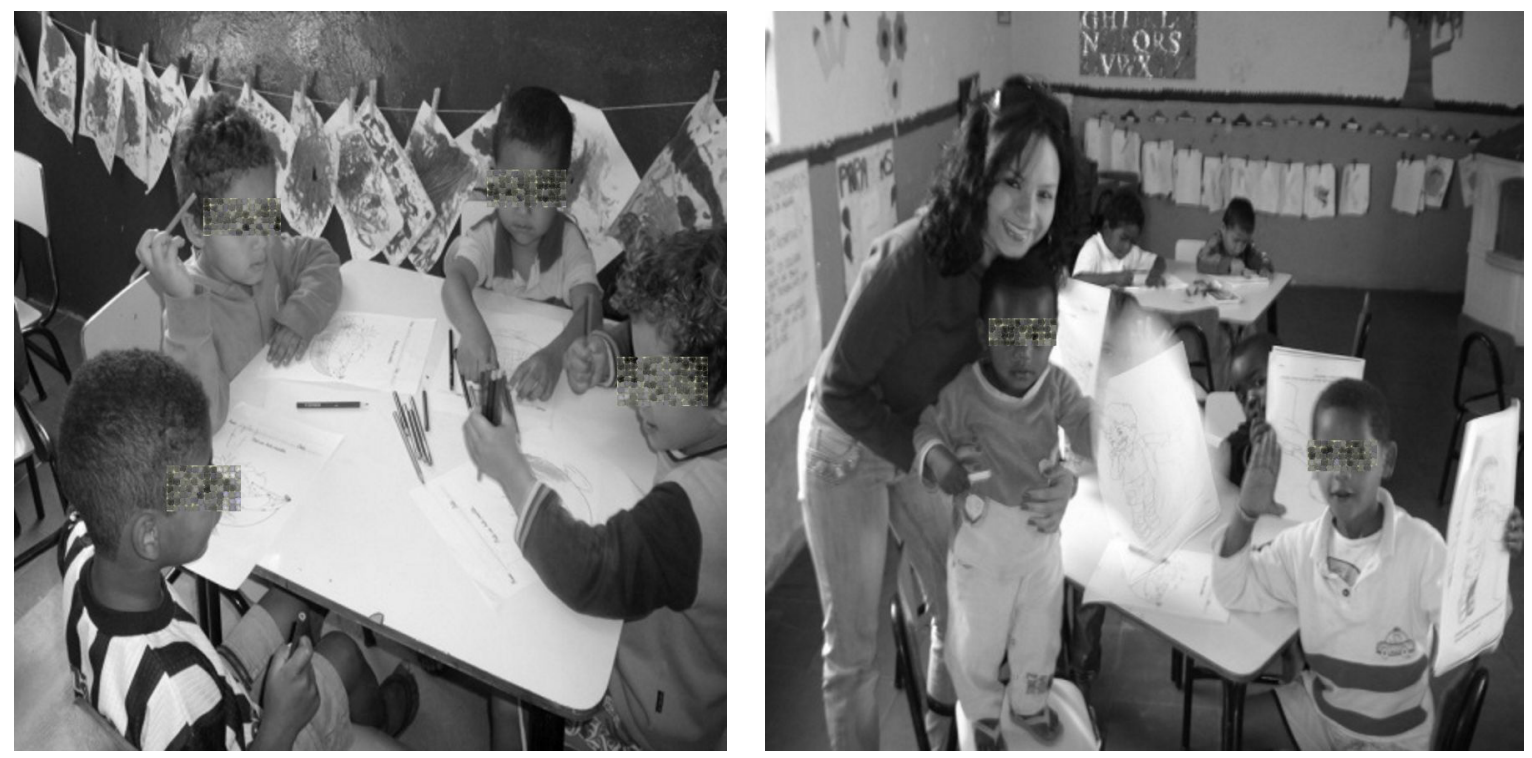

Fonte: Acervo das autoras.

As reuniões com os pais contaram com a participação de $59 \%(n=88)$ dos pais e responsáveis. Foi entregue, ao final da reunião, uma cartilha sobre a Pirâmide Alimentar para crianças, confeccionada pelos estudantes de nutrição, englobando dicas de alimentação saudável voltadas para a realidade do local e para a faixa etária pré-escolar.

O envolvimento dos pais nas atividades do estudo é de suma importância, pois a família e creche podem promover situações complementares e significativas de aprendizagem e convivência que vão ao encontro das necessidades e demandas das crianças das instituições. Mesmo havendo diferenças distintas entre as obrigações da família e as da creche, há também responsabilidades e objetivos comuns a ambas (EPSTEIN, 1987; HADDAD, 1987).

O curso de capacitação para manipuladores de alimentos contou com 58,33\% de participação $(n=12)$. Foi possível trocar conhecimento e fixação do aprendizado, bem como promover a melhoria dos hábitos de higiene e manuseio de alimentos. Foi possível, ainda, conscientizar os colaboradores em relação à importância de adotar boas práticas de higiene pessoal, manusear, preparar e porcionar corretamente os alimentos.

Em revisão bibliográfica realizada por Bellizzi e colaboradores acerca do treinamento de manipuladores de alimentos em UAN, no período de 1994 a 2000, foi identificado que as estratégias de ensino predominantes foram aulas expositivas, aliadas às atividades de dinâmicas de grupo, 0 que, em parte, assemelha-se à metodologia adotada no presente estudo (BELLIZZI et al., 2005).

Nesse contexto, cabe ressaltar a necessidade de programas de formação para estas profissionais, uma vez que são parte integrante da educação e contribuem no ambiente escolar para o processo educativo das crianças.

Acredita-se que para promover hábitos alimentares mais saudáveis e diminuir os índices de 
obesidade e carências nutricionais é necessário adquirir conhecimentos sobre alimentação e nutrição (COSTA et al., 2004; TRICHES; GIUGLIANI, 2005).

\section{CONSIDERAÇÕES FINAIS}

A educação nutricional é um processo ativo, que propõe levar a ciência da nutrição ao ser humano, obtendo-se mudanças de atitudes, práticas alimentares e de conhecimentos nutricionais como garantia da saúde do homem (BRASIL, 2013).

Os trabalhos desenvolvidos proporcionaram, por meio da educação nutricional, a melhoria do estado nutricional dos pré-escolares dos Núcleos Comunitários participantes da pesquisa. Notouse o fortalecimento da promoção de mudanças com as atividades lúdicas, buscando a instalação de hábitos alimentares saudáveis, flexibilização das ações humanas na atuação profissional, desenvolvimento de novos potenciais e impulsionamento da criatividade no exercício do cuidar.

\section{REFERÊNCIAS}

ALBIERO, K. A.; ALVES, F. S. Formação e desenvolvimento de hábitos alimentares em crianças pela educação nutricional. Revista Nutrição em Pauta, São Paulo, v. 15, n. 82, p. 17-21, jan./ fev, 2007.

ANTONIO, M. A. M. et al. Avaliação nutricional das crianças matriculadas nas quatorze creches municipais de Paulínia-SP. Revista Paulista de Pediatria, São Paulo, v. 1, p. 12-15, 1996.

AZEVEDO, U. N. D. et al. Humanização: um olhar integral das práticas de saúde. In: CONGRESSO BRASILEIRO DE MEDICINA DE FAMÍLIA E COMUNIDADE (CBMFC), 12., 2013, Belém. Anais... Rio de Janeiro: SBMFC, 2013, p. 1497.

BARBOSA, R. M. S.; SALLES-COSTA, R.; SOARES, E. A. Guias alimentares para crianças: aspectos históricos e evolução. Rev. Nutr., Campinas, v. 19, n. 2, p. 255-263, mar./abr. 2006.

BARROS, F. C.; VICTORA, C. G.; VAUGHAN, J. P. The Pelotas birth cohort study (1982-1987): strategies for following up 6,000 children in a developing country. Paediatric and Perinatal Epidemiology, New Jersey, v. 4, p. 267-282, june 2008.

BELLIZZI, A. et al. Treinamento de manipuladores de alimentos: uma revisão de literatura. Revista Higiene Alimentar, Mirandópolis, v. 19, n. 133, p. 36-48, 2005.

BRASIL, E. et al. Educação alimentar e nutricional como ferramenta efetiva de saúde do escolar: uma revisão de literatura. FIEP Bulletin On-line, Foz do Iguaçu, v. 83, n. 2, 2013.

CORSO, A. C.; VITERITTE, P. L.; PERES, M. A. T. Prevalência de sobrepeso e sua associação com a área de residência em crianças menores de 6 anos de idade matriculadas em creches 
públicas de Florianópolis, Santa Catarina, Brasil. Rev. Bras. Epidemiol., São Paulo, v. 7, n. 2, p. 201-208, jun. 2004.

COSTA, A. G. V. et al. Aplicação de jogo educativo para a promoção da educação nutricional de crianças e adolescentes. Revista Nutrição Brasil, São Paulo, v. 3, n. 4, p. 205-209, jul./ago. 2004.

EPSTEIN, J. Toward a theory of family-school connections: teacher practices and parent involvement. In: HURRELMANN. K.; KAUFMANN, F.; LOSEL, F. (Ed.). Social intervention: potential and constraints. Berlin: Mouton de Gruyter, 1987.

FAGIOLI, D.; NASSER, L. A. Educação nutricional na infância e na adolescência: planejamento, intervenção, avaliação e dinâmica. São Paulo: RCN, 2006.

BRASIL. Ministério da Educação. Fundo Nacional de Desenvolvimento da Educação (FNDE). Resolução $\mathrm{n}^{\mathrm{o}}$ 32, de 10 de agosto de 2006. Estabelece normas para a execução do Programa Nacional de Alimentação Escolar - PNAE. Brasília, DF: MEC, 2006.

GIUGLIANI, E. R. J.; VICTORA, C. G. Alimentação complementar. J. Pediatr., Rio de Janeiro, v. 76, suppl. 3, p. 253-262, 2000.

HADDAD, L.. A relação creche-família: relato de uma experiência. Cadernos de Pesquisa, São Paulo, v. 60, p. 70-78, fev. 1987

JELLIFFE, D. B. The assessment of the nutritional status of the community. Geneva: WHO, 1966 (Monograph Series).

LIMA, D. B. et al. Crescendo com saúde e nutrição: aplicação do lúdico na educação nutricional. Em Extensão, Uberlândia, v. 8, n. 2, p. 59-67, jun./dez. 2009.

MIRANDA, A. S. et al . Anemia ferropriva e estado nutricional de crianças com idade de 12 a 60 meses do município de Viçosa, MG. Rev. Nutr., Campinas, v. 16, n. 2, p. 163-169, jun. 2003.

MONTEIRO, C. A.; CONDE, W. L. Evolução da obesidade nos anos 90: a trajetória da enfermidade segundo estratos sociais no nordeste e sudeste do brasil. In: MONTEIRO, C. A. (Org.). Velhos e novos males da saúde no Brasil: a evolução do país e suas doenças. São Paulo: EDUSP, 2000.

MONTEIRO, E. A. A. et al. Resgate da concepção criativa e humanizada no processo pedagógico da educação nutricional. Revista Brasileira de Nutrição Clínica, São Paulo, v. 23, n. 2, p. 51 $55,2008$.

OLIVEIRA, M. C. F.; SILVA, M. M. S.; SANT’ANA, M. S. L. Alimentação saudável do préescolar. In: SILVA, M. M. S.; CAMPOS, M. T. F. S. (Org.). Segurança alimentar e nutricional na atenção básica em saúde. Viçosa, MG: UFV, 2003.

QUEIROZ, D. Deficiência de vitamina A em crianças de 6 a 59 meses de idade na área urbana do estado da Paraíba. 2008. 83 f. Dissertação (Mestrado) - Universidade Estadual da Paraíba, Campina Grande, 2008. 
RIBAS, D. L. B. et al. Saúde e estado nutricional infantil de uma população da região CentroOeste do Brasil. Rev. Saúde Pública, São Paulo, v. 33, n. 4, p. 358, 365, ago. 1999.

SCHMITZ, B. A. S. et al. A escola promovendo hábitos alimentares saudáveis: uma proposta metodológica de capacitação para educadores e donos de cantina escolar. Cad. Saúde Pública, Rio de Janeiro, v. 24, suppl. 2, p. 312-322, 2008.

SMELTZER, S.; BARE, B. Tratamento de enfermagem médico-cirúrgica. Rio de Janeiro: Guanabara Koogan, 2005.

TUMA, R. C. F. B.; COSTA, T. H. M.; SCHMITZ, B. A. S. Avaliação antropométrica e dietética de pré-escolares em três creches de Brasília, DF. Rev. Bras. Saúde Matern. Infant., Recife, v. 5, n. 4, p. 419-428, out./dez. 2005.

TRICHES, R. M.; GIUGLIANI, E. R. J. Obesidade, práticas alimentares e conhecimentos de nutrição em escolares. Revista de Saúde Pública, São Paulo, v. 39, n. 4, p. 541-547, ago. 2005.

VITOLO, M. R. et al. Impactos da implementação dos dez passos da alimentação saudável para crianças: ensaio de campo randomizado. Cad. Saúde Pública, Rio de Janeiro, v. 21, n. 5, p. 1448-1457, set.-out. 2005.

WHO. Beta version feb 17th, 2006: software for assessing growth and development of the world's children. 2006. Disponível em: <http://www.who.int/childgrowth/software/en>. Acesso em: 11 set. 2012.

WHO. WHO reference 2007: growth reference data for 5-19 years. Disponível em: <http:// www.who.int/growthref/en/ 2007>. Acesso em: 11 set. 2012.

ZAMBOM, M. P. et al. Correlação entre o índice de massa corporal e a prega cutânea tricipital em crianças da cidade de Paulínia, SP. Rev. Assoc. Med. Bras., São Paulo, v. 49, n. 2, p. 137 140, abr./jun. 2003.

Submetido em 5 de abril de 2013.

Aprovado em 4 de junho de 2013. 